\section{Acesso a e utilização de serviços odontológicos no Estado do Rio de Janeiro, Brasil, em 1998: um estudo exploratório a partir da Pesquisa Nacional por Amostra de Domicílios}

\author{
Access to and utilization of dental services in \\ the State of Rio de Janeiro, Brazil: an exploratory \\ study based on the 1998 National Household \\ Sample Survey
}

\author{
${ }^{I}$ Núcleo de Estudos de \\ Saúde Coletiva, Universidade \\ Federal do Rio de Janeiro, \\ Rio de Janeiro, Brasil. \\ Correspondência \\ A. L. D. Manhães \\ Núcleo de Estudos de Saúde \\ Coletiva, Universidade \\ Federal do Rio de Janeiro. \\ Praça da Prefeitura \\ Universitária $s / n$ \\ Rio de Janeiro, $R J$ \\ 21949-900, Brasil. \\ aldmanhaes@bol.com.br
}

\begin{abstract}
This study focused on access to and utilization of dental services in the State of Rio de Janeiro, Brazil, based on data from the 1998 National Household Sample Survey. The study population included 7,756 individuals, stratified by age (15 to 19, 35 to 44, and 65 to 74 years). Measurement of access to dental services was based on answers to the question regarding the last visit to a dentist. The answer "never visited a dentist" was interpreted as lack of access and was analyzed according to demographic, socioeconomic, and epidemiological variables. The proportions of individuals who had never visited a dentist were $7.6 \%, 1.8 \%$, e 2.6\%, respectively for young people, young adults, and the elderly. The results suggest that low socioeconomic status was associated with lack of access to dental services, characterized by low schooling (youth and young adults), low per capita income (youth and the elderly), and family wealth as measured by number of home appliances. Health insurance coverage was inversely associated with lack of access among youth and young adults.
\end{abstract}

Dental Care; Health Services Accessibility; Health Inequalities
Antonio Luís Dias Manhães ${ }^{1}$

Antonio José Leal Costa 1

\section{Introdução}

O conceito de acesso a serviços de saúde é, ainda hoje, alvo de debate e discussão 1,2. Tem o significado amplo de representar o grau de adequação entre o cliente e o sistema de saúde. Implica garantia de ingresso no sistema de saúde sem obstáculos físicos, financeiros ou de outra natureza. É um conceito complexo, muitas vezes empregado de forma imprecisa e pouco clara na sua relação com o uso de serviços de saúde. A definição de acesso varia entre autores, ao longo do tempo e de acordo com o contexto em que é empregada 3 .

$\mathrm{O}$ acesso aos serviços permeia as relações entre as necessidades de saúde, a demanda por e a utilização de serviços de saúde. Estes três elementos, quando organizados seqüencialmente, indicam que as "necessidades" das pessoas em termos de saúde transformam-se em "demanda" que, por sua vez, gera a "utilização" dos serviços, desde que garantido o acesso a eles 4 .

Diversos trabalhos realizados em países desenvolvidos utilizaram o modelo proposto por Andersen \& Newman 5, em 1973, para estudar os determinantes do uso de serviços de saúde enquanto medida de acesso, inicialmente na avaliação de serviços médicos e, mais recentemente, na de serviços odontológicos 6,7,8,9,10,11. Neste modelo, três tipos de características são considerados para explicar as diferenças no uso de serviços de saúde: predisposição, facilitação e 
necessidade. No primeiro tipo, representando a predisposição do indivíduo para usar os serviços de saúde, incluem-se variáveis sócio-demográficas (idade, sexo, estado civil, tamanho da família, etnia, escolaridade e ocupação) e atitudes e opiniões do indivíduo (valores em relação à saúde e conhecimento sobre as doenças). No segundo, incluem-se variáveis que facilitam o uso desses serviços, tais como renda familiar, existência ou não de plano de saúde e acesso a fonte regular de cuidado dentário. Compõem o terceiro tipo, representando a necessidade do uso de serviços de saúde, a percepção do estado de saúde pelo indivíduo ou o diagnóstico da doença pelo profissional de saúde. Os resultados desses e de outros estudos 12,13,14,15 mostram que pessoas do sexo feminino, com idade entre 5 e 24 anos, com maiores renda e escolaridade têm mais acesso e utilizam os serviços odontológicos com mais freqüência e, ou, de forma mais regular.

No Brasil, sabe-se que boa parte da população não tem acesso aos serviços de saúde odontológicos, como mostram os resultados dos últimos inquéritos sobre saúde bucal de abrangência nacional 16 . Bertoldi \& Barros 17 constataram a existência de iniqüidades no acesso e na utilização dos serviços odontológicos no Brasil, a partir dos dados do suplemento de saúde da Pesquisa Nacional por Amostra de Domicílios (PNAD) de 199818.

Segundo o Projeto SB Brasil 2003 19, cerca de $14 \%$ dos adolescentes brasileiros nunca foram ao dentista. Os idosos brasileiros, por sua vez, apresentam uma baixa utilização de serviços odontológicos. Em seu conjunto, a proporção de idosos que visitaram o dentista há menos de um ano em $2003(13,2 \%)$ foi cerca de três vezes menor que a observada na população idosa americana 20,21,22 . Também chama a atenção a proporção de idosos que jamais visitaram um dentista em nosso país $(6,3 \%)$, representando um contingente de cerca de 800 mil indivíduos 23 .

A oferta de serviços odontológicos no Estado do Rio de Janeiro, Brasil, é adequada e acima dos padrões, quando avaliada por meio da relação dentista/população, da ordem de um cirurgião dentista para cada 607 habitantes, a segunda maior razão em todo o Brasil, ficando atrás apenas do Estado de São Paulo (Conselho Federal de Odontologia. http://www.cfo.org. br/busca_dados/totais/tot_prof_cro.asp, acessado em 15/Abr/2005). Não obstante, supõe-se que, tal como no âmbito nacional, os padrões de acesso e utilização de serviços odontológicos no Estado do Rio de Janeiro sejam heterogêneos e estejam associados a fatores de natureza social, econômica, cultural e demográfica, e também intermediados pela oferta de serviços públicos odontológicos.
O presente estudo teve como objetivo analisar o acesso e a utilização de serviços odontológicos no Estado do Rio de Janeiro, a partir dos dados constantes na base do suplemento de saúde da PNAD 1998 18. Busca-se assim contribuir para a identificação de desigualdades, com vistas a subsidiar o adequado planejamento de ações de saúde bucal, em consonância com as estratégias para alcançar as metas estabelecidas pela Organização Mundial da Saúde (OMS) para o ano 202024

\section{Materiais e métodos}

Utilizou-se a base de dados da PNAD 1998, realizada pelo Instituto Brasileiro de Geografia e Estatística (IBGE). O corpo básico da PNAD inclui informações sobre características do domicílio e de seus moradores. No ano de 1998, o Ministério da Saúde patrocinou a inclusão de um módulo suplementar, para obter informações atualizadas e de abrangência nacional sobre acesso a e utilização de serviços de saúde, cobertura por diferentes modalidades de planos de saúde, necessidades de saúde em função da existência de doenças crônicas, limitação de atividades habituais por problemas de saúde crônicos ou agudos e gastos com planos privados de saúde, serviços e medicamentos 18 .

A PNAD 1998 tem cobertura nacional, com representatividade estadual e de nove regiões metropolitanas. Trata-se de um estudo seccional, cuja amostra foi obtida por um processo de seleção de domicílios em três estágios: municípios (unidade amostral primária), setores censitários e domicílios. Para o processo de amostragem foi utilizada a população e a malha de setores do Censo Demográfico de 199118.

Ao todo, foram entrevistadas 26.212 pessoas residentes no Estado do Rio de Janeiro por meio de um questionário estruturado. Deste contingente extraiu-se a população de estudo, composta por três estratos formados pelos indivíduos que, no momento da realização da PNAD 1998, apresentavam idades nas faixas dos 15 aos 19 anos (2.429 pessoas), dos 35 aos 44 anos (3.999 pessoas) e dos 65 aos 74 anos (1.328 pessoas), respectivamente. A opção por tais faixas etárias foi feita em consonância com as recomendações da OMS para a realização de estudos populacionais sobre saúde oral 25,26.

A seleção da faixa dos 15 aos 19 anos deveuse ao fato de, na idade de 15 anos, os dentes permanentes já terem sido expostos ao ambiente bucal por 3 a 9 anos. Esta idade também é importante para a avaliação dos indicadores de doenças periodontais em adolescentes. A ava- 
liação aos 18 anos é importante para se ter uma representação do perfil das doenças bucais em adultos jovens e, além disso, esta idade é referência para avaliação das condições de saúde bucal em nível internacional, explicitada nas metas da OMS/Federation Dentaire Internationale (FDI) para o ano 200025.

A faixa etária de 35 a 44 anos é o grupo padrão para avaliação das condições de saúde bucal em adultos. O efeito total da cárie dentária, o nível de gravidade do envolvimento periodontal e os efeitos gerais do tratamento prestado podem ser monitorados usando-se dados deste grupo etário.

O grupo etário dos 65 a 74 anos tem se tornado mais importante com as mudanças na distribuição etária e o aumento da expectativa de vida que vem ocorrendo em todos os países. Os dados deste grupo são necessários tanto para o planejamento adequado do tratamento destinado aos idosos como para o monitoramento dos efeitos gerais dos serviços odontológicos prestados a uma população 25 .

A avaliação do acesso a serviços odontológicos baseou-se na variável $v 1349$ do banco de dados da PNAD 1998, relativa à pergunta sobre $\mathrm{a}$ última vez que o entrevistado foi ao dentista. No questionário da PNAD 1998 havia quatro respostas possíveis a esta pergunta "Quando (...) foi ao dentista pela última vez?”, a saber: (a) “menos de 1 ano"; (b) "de 1 ano a 2 anos"; (c) "3 ou mais anos"; e (d) "nunca foi ao dentista". Partiu-se da premissa de que, ao longo da vida, de maneira geral a grande maioria das pessoas se vêem, em algum momento, com alguma necessidade de atendimento odontológico, por diferentes motivos. O fato de um indivíduo nunca ter ido ao dentista na vida, portanto, seria indicativo de restrição do acesso a serviços odontológicos, principalmente quando referido a idades correspondentes a tempos de exposição ao risco de desenvolvimento de problemas de saúde bucal suficientemente longos, como para as faixas etárias selecionadas. De acordo com a resposta, os participantes foram então classificados em dois grupos: aqueles que visitaram o dentista alguma vez na vida e aqueles que nunca visitaram o dentista. Em seguida, estimou-se para cada grupo de idade a proporção de indivíduos que responderam nunca terem ido ao dentista até o momento da entrevista, como indicador da limitação de acesso a serviços de saúde odontológicos.

Além da idade (variável de estratificação), os padrões de acesso a serviços odontológicos foram analisados segundo categorias de variáveis demográficas, epidemiológicas e sócio-econômicas. As variáveis de natureza demográfica foram sexo (masculino e feminino) e área de residência (urbana e rural). A variável de cunho epidemioló- gico selecionada foi a avaliação do próprio estado de saúde, agrupada nas categorias satisfatória e não satisfatória a partir das respostas muito bom ou bom e regular, ruim ou muito ruim à pergunta "De um modo geral (...) considera o seu próprio estado de saúde como:”, respectivamente.

Já as características sócio-econômicas foram representadas pelas variáveis escolaridade (até 3 anos, de 4 a 7 anos, 8 ou mais anos de estudo), renda mensal per capita (inferior a um salário mínimo - SM, igual ou superior a um SM e inferior a dois $\mathrm{SM}$, igual ou superior a dois salários mínimos), classe econômica (baixa, média e alta) e ter plano de saúde (sim ou não). A renda per capita foi calculada dividindo-se a renda domiciliar informada pelo número de residentes no domicílio, tendo como unidade de referência o valor do SM da época (R\$ 130,00). As categorias de classe econômica baixa, média e alta foram estabelecidas em função da posse informada de diferentes bens de consumo, a saber: menos do que três bens básicos (rádio, televisão e geladeira), menos de três bens básicos e um adicional (freezer ou máquina de lavar) e possuir bens adicionais e um ou mais bens básicos, respectivamente.

Por fim, foi incluída na análise a variável informante (o próprio ou outra pessoa, moradora ou não no domicílio), com o intuito de verificar a possibilidade de ocorrência de viés de informação.

Na PNAD 1998, a coleta dos dados relativos às variáveis de natureza demográfica e sócio-econômica - exceto a posse de plano de saúde - teve como datas e períodos de referência o dia 26 de setembro, a semana de 20 a 26 de setembro ou o mês de setembro de 1998. Por sua vez, para as variáveis: avaliação do próprio estado de saúde, ter ou não plano de saúde e última vez que foi ao dentista, a referência foi a data da realização da entrevista 18 .

A análise dos dados consistiu, inicialmente, na descrição dos três estratos etários quanto às categorias das variáveis independentes selecionadas. A existência de associação entre restrição do acesso aos serviços odontológicos e as variáveis independentes, dentro de cada estrato etário, foi verificada em duas etapas. Na primeira, foram calculadas razões de chances (ou odds ratios - OR) brutas e seus respectivos intervalos de $95 \%$ de confiança (IC95\%), obtidos pelo método de Woolf. A segunda etapa consistiu na análise por meio de modelos de regressão logística múltipla, os quais geraram estimativas das OR ajustadas e seus respectivos IC95\%. Para inclusão das variáveis independentes em tais modelos, foram selecionadas aquelas cujos resultados das análises bivariadas apresentaram significância estatística expressa por valores de $\mathrm{p}$ iguais ou menores que 
0,20 , obtidos por meio do teste do qui-quadrado. Uma vez incluídas nos modelos múltiplos, as variáveis independentes foram retiradas passo a passo, mantendo-se, ao final, somente aquelas que apresentaram significância estatística no nível de 5\% ( $<$ <,05). A única exceção foi a variável informante, cuja manutenção nos três modelos finais foi definida a priori, tendo em vista a possibilidade de introdução de viés de informação, como já mencionado. $\mathrm{O}$ ajuste dos modelos finais foi verificado por meio do teste de Hosmer \& Lemeshow 27.

Tendo em vista o plano amostral da PNAD, utilizou-se o peso amostral relativo a cada indivíduo selecionado (correspondente ao inverso da probabilidade de seleção), ajustado pelo peso amostral médio referente ao conjunto dos residentes no Estado do Rio de Janeiro então selecionados. Buscou-se assim, resgatar, na população de estudo, a distribuição das variáveis selecionadas vigente na população do Estado do Rio de Janeiro em 1998, preservando o tamanho da amostra 28.

\section{Resultados}

As proporções de indivíduos que responderam nunca ter ido ao dentista na vida foram $7,6 \%$, $1,8 \%$ e 2,6\%, respectivamente para as faixas de 15 a 19,35 a 44 e 65 a 74 anos.

Observou-se uma elevação da proporção de mulheres à medida que aumentou a idade, correspondendo a $50,8 \%, 53 \%$ e $58,3 \%$ entre os jovens, os adultos e os idosos, respectivamente (Tabela 1). Da mesma forma, as proporções da população com renda mais elevada (2 ou mais $\mathrm{SM})$ e que responderam às perguntas da entrevista por si próprias também aumentaram à medida que a idade se elevou.

Nas três faixas etárias analisadas, aproximadamente dois entre cada três indivíduos foram classificados como pertencendo à classe econômica média.

A proporção da população com escolaridade mais elevada (8 ou mais anos de estudo) diminuiu à medida que a idade aumentou, passando de $46,8 \%$ na faixa etária dos jovens para $23,2 \%$ entre os idosos, uma redução de quase a metade em relação à primeira faixa etária.

Quanto à avaliação do próprio estado de saúde, percebe-se que a freqüência de avaliações positivas do próprio estado de saúde caiu sistematicamente à medida que a idade aumentou, chegando a atingir entre os idosos valor próximo da metade do observado entre os jovens (Tabela 1).

$\mathrm{Na}$ análise bivariada (Tabela 2) todas as variáveis mostraram-se de alguma forma associa- das ao desfecho (i.e., com OR diferentes de 1,0), porém com variações dos níveis de significância estatística. No grupo etário de 35 a 44 anos, o sexo masculino mostrou-se mais fortemente associado ao desfecho nunca ter ido ao dentista $(\mathrm{OR}=$ 2,34). Já na faixa etária de 65 a 74 anos, o sexo masculino mostrou-se inversamente associado ao desfecho $(\mathrm{OR}=0,91)$, porém sem significância estatística.

Com relação às variáveis de natureza sócioeconômica (renda per capita, escolaridade, classe econômica e plano de saúde), em todas as faixas etárias, à queda do padrão sócio-econômico correspondeu o aumento da privação de acesso a serviços odontológicos.

Nas três faixas etárias observou-se uma forte associação entre renda e o desfecho analisado, sob forma de gradiente, ou seja, à medida que diminuiu a renda, aumentou a OR de nunca ter ido ao dentista. Em particular, entre aqueles com renda per capita inferior a um salário mínimo, a chance de nunca ter ido ao dentista foi cerca de 4,5 vezes a observada entre os grupos com renda igual ou superior a dois salários, em todas as faixas de idade.

Quanto à escolaridade, a condição de analfabeto funcional ( 0 a 3 anos de estudo) mostrou-se fortemente associada com a privação de acesso a serviços odontológicos, particularmente na faixa dos 35 aos 44 anos, na qual a razão de chances $(\mathrm{OR}=11,2)$ equivaleu a quase o dobro da observada na primeira faixa etária. $\mathrm{O}$ fato de não ter sido selecionado nenhum indivíduo com oito ou mais anos de estudo e que tivesse relatado nunca ter ido ao dentista na faixa etária de 65 a 74 anos impossibilitou a adequada estimação da OR nessa faixa etária.

$\mathrm{Na}$ análise da classe econômica, entre os menos favorecidos (menos que três bens básicos) a chance de nunca ter ido ao dentista foi sempre mais elevada em relação aos mais favorecidos, sendo a OR mais expressiva próxima a 40, registrada na faixa etária de 35 a 44 anos. Tal associação, nesta faixa etária, foi cerca de sete vezes maior em relação às outras duas faixas de idade. Tal como com a renda per capita, as associações entre acesso a serviços odontológicos e as categorias de escolaridade e classe econômica mostraram-se sob a forma de gradientes (Tabela 2).

Não ter direito a plano de saúde esteve positivamente associado ao desfecho, com OR iguais a 5,58, 8,54 e 2,31 respectivamente para a primeira, segunda e terceira faixas etárias.

Somente na primeira faixa etária, a avaliação negativa do próprio estado de saúde mostrou-se associada à restrição de acesso a serviços odontológicos. Nas demais não se observou significância estatística. 
Distribuição (\%) da população de estudo segundo características demográficas, sócio-econômicas e epidemiológicas, por faixa etária. Estado do Rio de Janeiro, Brasil, 1998

\begin{tabular}{|c|c|c|c|}
\hline \multirow[t]{3}{*}{ Variáveis } & \multicolumn{3}{|c|}{ Faixa etária (anos) } \\
\hline & 15 a 19 & 35 a 44 & 65 a 74 \\
\hline & $(n=2.429)$ & $(n=3.999)$ & $(n=1.328)$ \\
\hline \multicolumn{4}{|l|}{ Sexo } \\
\hline Feminino & 50,8 & 53,0 & 58,3 \\
\hline Masculino & 49,2 & 47,0 & 41,7 \\
\hline \multicolumn{4}{|c|}{ Renda per capita (salários mínimos *) } \\
\hline$\geq 2$ & 26,1 & 37,8 & 47,9 \\
\hline$\geq 1 \mathrm{e}<2$ & 33,9 & 30,4 & 26,8 \\
\hline$<1$ & 40,0 & 31,8 & 25,3 \\
\hline \multicolumn{4}{|l|}{ Escolaridade (anos) } \\
\hline 8 ou + & 46,8 & 55,0 & 23,2 \\
\hline $4-7$ & 42,1 & 26,8 & 30,2 \\
\hline $0-3$ & 11,1 & 18,3 & 46,5 \\
\hline \multicolumn{4}{|l|}{ Classe econômica ** } \\
\hline Alta & 24,9 & 26,0 & 23,3 \\
\hline Média & 66,4 & 66,8 & 67,2 \\
\hline Baixa & 8,7 & 7,1 & 9,5 \\
\hline \multicolumn{4}{|l|}{ Tem direito a plano de saúde } \\
\hline $\operatorname{Sim}$ & 28,6 & 35,2 & 37,9 \\
\hline Não & 71,4 & 64,8 & 62,1 \\
\hline \multicolumn{4}{|l|}{ Avaliação do estado de saúde } \\
\hline Muito bom ou bom & 95,3 & 82,6 & 43,5 \\
\hline Regular, ruim ou muito ruim & 4,7 & 17,4 & 56,5 \\
\hline \multicolumn{4}{|l|}{ Situação do domicílio } \\
\hline Urbano & 94,5 & 95,7 & 96,3 \\
\hline Rural & 5,5 & 4,3 & 3,7 \\
\hline \multicolumn{4}{|l|}{ Informante } \\
\hline O próprio & 23,5 & 50,4 & 64,7 \\
\hline Outro & 76,5 & 49,6 & 35,3 \\
\hline
\end{tabular}

Fonte: Pesquisa Nacional por Amostra de Domicílios de 1998 (PNAD 1998).

* Salário mínimo: $\mathrm{R} \$ 130,00$.

** Classe econômica: alta - freezer e máquina de lavar roupas + um ou mais itens básicos (rádio, televisão e geladeira); média

- três bens básicos ou menos que três bens básicos e um adicional (freezer e máquina de lavar roupas); baixa - menos que

três bens básicos.

Por sua vez, quem tinha seu domicílio na zona rural apresentou chances mais elevadas de nunca ter ido ao dentista, no entanto, em nenhuma faixa etária os resultados apresentaram significância estatística.

Entre os registros cujas respostas foram obtidas por terceiros (outro informante), as chances de nunca ter ido ao dentista foram mais elevadas em relação àquelas relativas ao próprio informante, sendo que à medida que aumentou a idade, aumentaram as OR.

$\mathrm{Na}$ análise múltipla, uma vez ajustadas para o efeito de confundimento das demais variáveis independentes, as OR tenderam a apresentar valores inferiores, quando comparadas às respectivas OR brutas. Por vezes, junto com a queda das OR, observou-se também perda da significância estatística. Na Tabela 3 são apresentados os resultados dos modelos múltiplos finais relativos a cada faixa etária, nos quais foram mantidas apenas as variáveis que apresentaram significância estatística, exceto no caso da variável informante (faixa etária de 35 a 44 anos), conforme mencionado anteriormente.

Quanto à variável sexo, só houve significância estatística na segunda faixa etária, porém com a 
Razões de chances (odds ratio - OR) brutas de nunca ter ido ao dentista * por faixas etárias, segundo características demográficas, sócio-econômicas e epidemiológicas. Estado do Rio de Janeiro, Brasil, 1998.

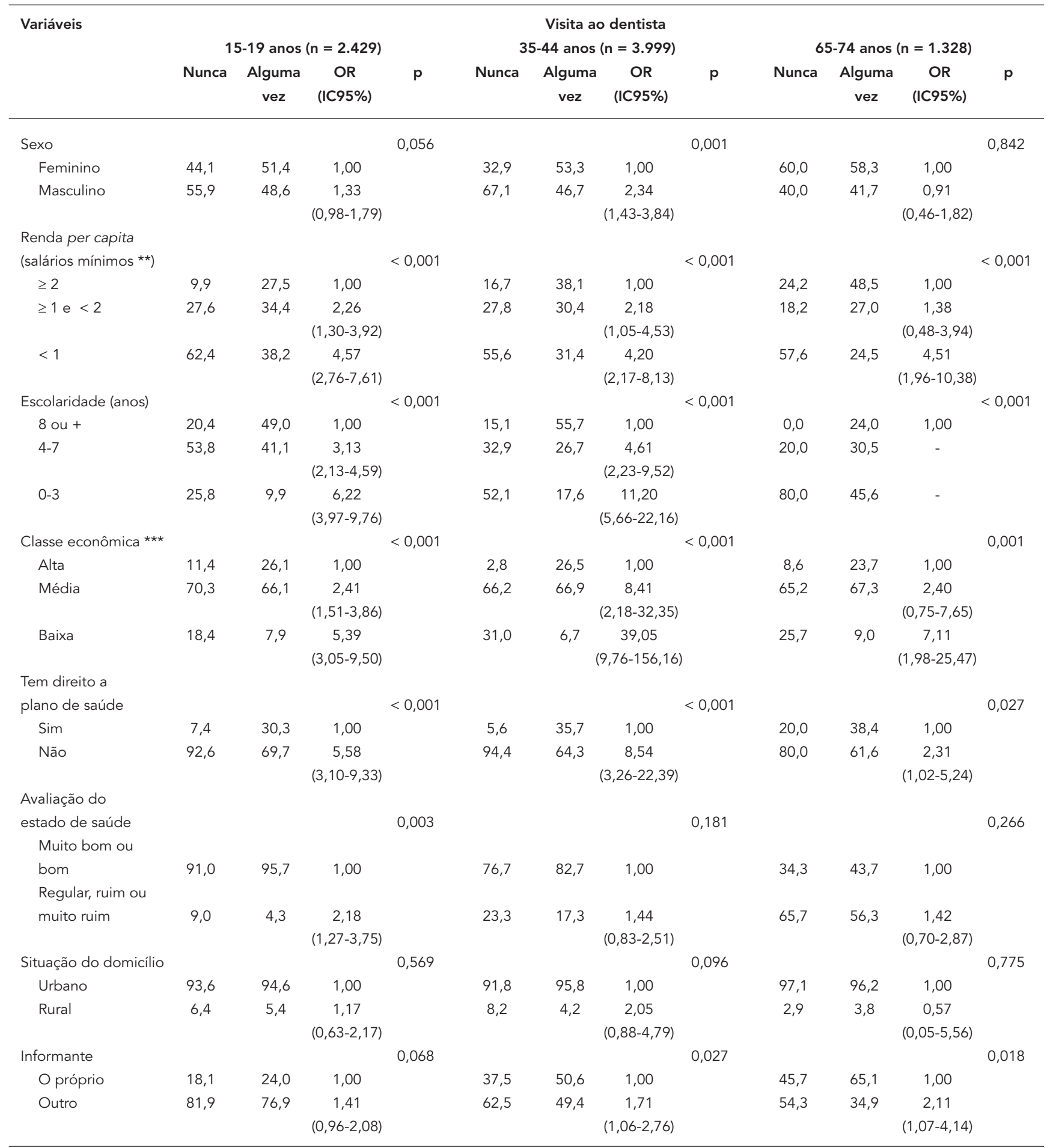

Fonte: Pesquisa Nacional por Amostra de Domicílios de 1998 (PNAD 1998).

p: teste do qui-quadrado de Pearson; OR (IC95\%): odds ratio não ajustada (intervalo de $95 \%$ de confiança).

* Obtidas por meio de regressão logística bivariada; categoria de referência: "nunca ter ido ao dentista";

** Salário mínimo: R\$130,00;

*** Classe econômica: alta - freezer e máquina de lavar roupas + um ou mais itens básicos (rádio, televisão e geladeira); média - três bens básicos ou menos que três bens básicos e um adicional (freezer e máquina de lavar roupas); baixa - menos que três bens básicos. 
Razões de chances (odds ratio - OR) ajustadas de nunca ter ido ao dentista * por faixas etárias, segundo características demográficas, sócio-econômicas e epidemiológicas. Estado do Rio de Janeiro, Brasil, 1998.

\begin{tabular}{|c|c|c|c|}
\hline \multirow[t]{3}{*}{ Variáveis } & \multicolumn{3}{|c|}{ Visita ao dentista (nunca vs. alguma vez) } \\
\hline & $15-19$ anos & $35-44$ anos & $65-74$ anos \\
\hline & OR (IC95\%) & OR (IC95\%) & OR (IC95\%) \\
\hline \multicolumn{4}{|l|}{ Sexo } \\
\hline Feminino & NS & 1,00 & NS \\
\hline Masculino & NS & $2,07(1,23-3,48)$ & NS \\
\hline \multicolumn{4}{|c|}{ Renda per capita (salários mínimos **) } \\
\hline$\geq 2$ & 1,00 & NS & 1,00 \\
\hline$\geq 1$ e $<2$ & $2,26(1,30-3,92)$ & NS & $1,36(0,47-3,88)$ \\
\hline$<1$ & $2,02(1,16-3,54)$ & NS & $4,72(2,04-10,89)$ \\
\hline \multicolumn{4}{|c|}{ Escolaridade (anos) } \\
\hline $8 \mathrm{ou}+$ & 1,00 & 1,00 & NS \\
\hline $4-7$ & $2,19(1,46-3,29)$ & $2,52(1,19-5,36)$ & NS \\
\hline $0-3$ & $3,96(2,46-6,37)$ & $4,92(2,36-10,23)$ & NS \\
\hline \multicolumn{4}{|c|}{ 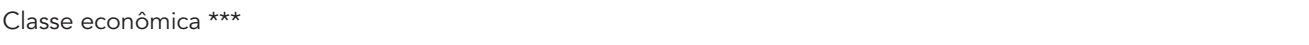 } \\
\hline Alta & NS & 1,00 & NS \\
\hline Média & NS & $3,94(1,00-15,54)$ & NS \\
\hline Baixa & NS & $11,85(2,82-49,74)$ & NS \\
\hline \multicolumn{4}{|c|}{ Tem direito a plano de saúde } \\
\hline Sim & 1,00 & 1,00 & NS \\
\hline Não & $2,71(1,50-4,92)$ & $2,79(1,01-7,70)$ & NS \\
\hline \multicolumn{4}{|l|}{ Informante } \\
\hline O próprio & 1,00 & 1,00 & 1,00 \\
\hline Outro & $1,53(1,03-2,28)$ & $1,55(0,93-2,59)$ & $2,13(1,05-4,28)$ \\
\hline
\end{tabular}

Fonte: Pesquisa Nacional por Amostra de Domicílios de 1998 (PNAD 1998).

OR (IC95\%): odds ratio ajustada (intervalo de 95\% de confiança); NS: não significativo do ponto de vista estatístico, no nível de $5 \%$ (excluída dos modelos finais), as variáveis avaliação do estado de saúde e situação do domicílio foram retiradas na medida em que os resultados não apresentaram significância estatística.

* Obtidas por meio de regressão logística bivariada; categoria de referência: "nunca ter ido ao dentista";

** Salário mínimo: $\mathrm{R} \$ 130,00$;

** Classe econômica: alta - freezer e máquina de lavar roupas + um ou mais itens básicos (rádio, televisão e geladeira); média - três bens básicos ou menos que três bens básicos e um adicional (freezer e máquina de lavar roupas); baixa - menos que três bens básicos.

magnitude da associação menor quando comparada à da respectiva OR não ajustada. A chance de ser do sexo masculino e nunca ter ido ao dentista foi cerca de duas vezes maior do que a observada entre as mulheres, na faixa etária de 35 a 44 anos.

A renda per capita manteve-se nos modelos múltiplos para as faixas etárias extremas. Na faixa dos 15 aos 19 anos o efeito dose resposta verificado na análise bivariada desapareceu. Assim, tanto entre aqueles com renda menor que $1 \mathrm{SM}$ quanto entre 1 e $2 \mathrm{SM}$, a chance de nunca ter ido ao dentista foi aproximadamente o dobro da observada entre aqueles pertencentes à faixa de renda per capita acima de 2 SM. Já na faixa de
65 a 74 anos observou-se um ligeiro aumento da magnitude da associação (único resultado de OR ajustado cuja magnitude não diminuiu em relação à medida não ajustada), ao se comparar a chance de nunca ter ido ao dentista entre as faixas de menor e maior renda. Não se observou significância estatística na segunda faixa de idade.

Quanto à escolaridade, observou-se significância estatística na primeira e segunda faixas etárias, porém com magnitude da associação menor quando comparada ao resultado não ajustado. Em relação aos mais escolarizados (8 ou mais anos de estudo), a chance dos analfabetos funcionais ( 0 a 3 anos estudo) nunca terem 
ido ao dentista foi cerca de quatro vezes maior entre 15 e 19 anos, aumentando para quase cinco vezes na faixa dos 35 aos 44 anos.

Quanto à classe econômica, somente na faixa de 35 a 44 anos observou-se significância estatística, com a magnitude da OR menor comparada à OR bruta (cerca de quatro vezes menor), porém ainda fortemente associado ao desfecho. Neste grupo etário, a chance de ser do grupo sócio-econômico menos favorecido (menos que três bens básicos) e nunca ter ido ao dentista foi mais de 11 vezes a do grupo de maior poder aquisitivo (freezer, máquina de lavar roupas e ao menos um bem básico). Tal como na análise bivariada, verificouse a presença de efeito dose resposta.

Não ter direito a plano de saúde manteve-se positivamente associado ao desfecho tanto na primeira como na segunda faixa etária, com as OR situando-se em torno de 2,7.

Com relação à variável informante, a chance de nunca ter ido ao dentista quando a resposta foi fornecida por terceiros foi cerca de 1,5 a 2,0 vezes mais elevada do que quando a resposta foi dada pelo próprio entrevistado. Apenas na faixa dos 35 aos 44 anos esta variável foi mantida, mesmo não apresentando significância estatística. Ressaltase que na análise bivariada, na faixa etária 15 a 19 anos, não se observou significância estatística no nível de $5 \%$, porém, na análise múltipla passou a apresentá-la, ocorrendo o inverso na segunda faixa de idade.

As OR relativas às variáveis relacionadas à avaliação do próprio estado de saúde e à situação do domicílio não apresentaram significância estatística em nenhuma faixa etária, sendo as mesmas excluídas dos modelos finais.

Os três modelos múltiplos apresentados mostraram-se satisfatoriamente ajustados aos respectivos dados de origem, tal como demonstrado por meio do teste de Hosmer \& Lemeshow 27, cujos valores de p para as faixas de 15 a 19 anos, 35 a 44 anos e 65 a 74 anos foram, respectivamente, $0,133,0,506$ e 0,642 .

\section{Discussão}

Na análise bivariada, com exceção da situação do domicílio, todas as variáveis mostraram-se associadas ao desfecho nunca ter ido ao dentista, considerado como privação de acesso, em pelo menos uma das três faixas etárias, ainda que com magnitudes variáveis. Na análise múltipla, as OR ajustadas mostraram-se menores que os seus correspondentes valores não ajustados, sendo a única exceção a variável renda per capita na faixa etária de 65 a 74 anos, para a qual a OR ajustada foi ligeiramente maior em relação à OR bruta.
Após o ajuste das OR nas análises múltiplas, algumas variáveis não apresentaram significância estatística e foram, portanto, excluídas dos modelos finais. Na faixa etária dos idosos, permaneceram as variáveis faixa de salário mínimo per capita e informante. Nas outras duas faixas de idade, mantiveram-se nos modelos finais as variáveis escolaridade, plano de saúde e informante, esta última mantida entre os adultos (35 a 44 anos) mesmo sem apresentar significância estatística. Além destas, mostraram-se associadas ao desfecho as variáveis renda per capita na faixa dos 15 aos 19 anos, e classe econômica e sexo, no grupo de idades entre 35 e 44 anos.

Tais achados são coerentes com os de outros estudos que permitem caracterizar a natureza essencialmente privada da assistência odontológica no Brasil: aproximadamente três em cada quatro indivíduos que demandaram assistência à saúde oral nas duas semanas que antecederam a realização das entrevistas na PNAD 1998 o fizeram em serviços privados 17 .

Investigações realizadas no Brasil mostram que a condição social tem sido enfatizada nas últimas décadas como importante determinante do uso de serviços odontológicos 29,30,31. No estudo desenvolvido na cidade de Bambuí, Minas Gearis, Matos et al. 32 verificaram que maior escolaridade e maior renda estavam positivamente associadas à visita regular ao dentista. Segundo dados da PNAD 1998, cerca de $20 \%$ dos brasileiros (29 milhões) nunca haviam consultado um dentista, com claras diferenças em relação à renda, região e localidade geográfica, chegando a $32 \%$ na área rural, apresentando, em sua maioria, renda de até dois salários mínimos 17. Em um estudo sobre uso de serviços odontológicos entre os estados do Brasil, baseado na PNAD 2003, os resultados mostraram que, tal como observado no Estado do Rio de Janeiro em 1998, as chances de uma pessoa nunca ter ido ao dentista foram menores entre aqueles com maior escolaridade e renda, e entre os que possuíam planos de saúde 33 .

Em países desenvolvidos, a situação sócioeconômica e/ou a escolaridade também mostraram-se positivamente associadas ao acesso e uso de serviços odontológicos entre jovens e idosos 20,34.

Segundo Frenk 2 , o conceito de acessibilidade fundamenta-se na noção de complementaridade entre características da oferta de serviços e da população. A acessibilidade é resultante da relação funcional entre os obstáculos à busca e obtenção de assistência (resistência) e a capacidade da população de superá-los (poder de utilização). As associações positivas entre renda e, ou escolaridade, e o acesso a serviços odontológicos 
por ora observadas sugerem, à luz do modelo teórico de Frenk 2, que os poderes dos usuários relacionados aos capitais econômicos e culturais podem significar maior capacidade de superação de obstáculos à busca e obtenção de assistência. Estes últimos estariam representados, por exemplo, pela natureza preponderantemente privada dos serviços odontológicos no Brasil 17.

$\mathrm{O}$ presente trabalho mostrou que, entre os homens, a chance de usar o serviço odontológico foi cerca da metade da observada entre as mulheres na faixa de 35 a 44 anos, sugerindo que jamais ter ido ao dentista esteve positiva e independentemente associado ao sexo masculino. Resultado semelhante foi observado com base nos dados da PNAD 2003 para todo o Brasil 33. Tais achados estão de acordo com o modelo de utilização de serviços de saúde desenvolvido por Andersen \& Newman 5 , no qual se considera que a influência do acesso no uso de serviços de saúde é função de fatores predisponentes, existentes previamente ao surgimento do problema de saúde e que afetam a predisposição das pessoas para usar serviços de saúde. Por exemplo, o gênero, já que as mulheres tendem a mostrar maior predisposição para o uso de serviços de saúde do que os homens 35.

Por ser o presente trabalho baseado em um estudo seccional, não é possível estabelecer com segurança a relação temporal entre as variáveis analisadas, limitando a identificação de associações de causa e efeito. Cabe considerar também a possível presença de viés de seleção, em particular o viés de sobrevivência seletiva. Supõe-se que, se presente, tal viés tenderia a enfraquecer a magnitude das associações observadas, principalmente no que tange às variáveis de natureza sócio-econômica.

Por sua vez, os resultados apresentados sugerem a presença de viés do informante. Via de regra, a chance de nunca ter ido ao dentista foi sempre mais elevada quando a resposta foi obtida a partir de terceiros, independentemente da faixa etária e das demais variáveis consideradas. Entretanto, a retirada da variável informante dos modelos finais não alterou os resultados observados quando da sua inclusão. Tais achados, coerentes com os observados a partir da análise de dados de inquéritos nacionais de saúde 36 , apontam para a necessidade da realização de estudos específicos, voltados para a caracterização e dimensionamento de tal viés, tendo em vista as elevadas freqüências de respostas obtidas a partir de terceiros na PNAD 1998.

Estudos conduzidos na Noruega, Canadá e também na Inglaterra 37 mostram que a disponibilidade e a facilidade de acesso a serviços odontológicos em certas regiões, bem como a distri- buição geográfica dos cirurgiões dentistas (razão dentista/população), interferem nos padrões de acesso e uso de serviços odontológicos 38,39. Considerando o padrão vigente no Estado do Rio de Janeiro, onde há aproximadamente um dentista para cada 600 habitantes, a restrição do acesso a serviços de saúde odontológicos parece estar mais fortemente associada a desigualdades de natureza sócio-econômica do que a limitações da oferta de tais serviços. Não obstante, a importância das variações regionais da oferta de serviços e programas de saúde oral nos âmbitos estadual e municipal deve ser alvo de investigações mais detalhadas.

Crianças brasileiras de 12 anos de idade e adolescentes de 15 a 19 anos apresentaram, em 2003 19, respectivamente, em média 2,8 e 6,2 dentes com experiência de cárie dentária. No que se refere a adultos, o número de dentes permanentes cariados, perdidos ou obturados devido à cárie (índice CPOD) foi, em média, 20,1 na faixa etária de 35 a 44 anos e 27,8 na de 65 a 74. Destaca-se o fato de que o componente perdido (P) foi responsável por cerca de $66 \%$ do índice no grupo de 35 a 44 anos e quase $93 \%$ no grupo de 65 a 74 anos. Tais níveis de morbidade vigentes em nossa população parecem sustentar a premissa inicial do presente estudo, de que, ao longo da vida, de maneira geral a grande maioria das pessoas se vêem, em algum momento, com alguma necessidade de atendimento odontológico, por diferentes motivos e, quando o fazem, a depender da condição sócio-econômica e inserção produtiva, o desfecho é quase sempre mutilador.

A opção por uma definição operacional mais específica de restrição de acesso, além de reduzir a freqüência de resultados falsos positivos, tem a vantagem de evitar as polêmicas relacionadas à caracterização de padrões ótimos de assistência odontológica. Se ainda não há consenso acerca da freqüência ideal de consultas odontológicas per capita num dado intervalo de tempo ${ }^{40}$, situações caracterizadas por subgrupos populacionais que nunca foram ao dentista podem ser seguramente interpretadas como indesejáveis, tendo em vista os níveis de morbidade vigentes em nosso país anteriormente citados.

No Estado do Rio de Janeiro em 1998, os contingentes populacionais que nunca utilizaram serviços odontológicos na vida corresponderam a 7,6\%; 1,8\% e 2,6\%, respectivamente para as faixas de 15 a 19 anos, 35 a 44 anos e 65 a 74 anos. Embora representando parcelas aparentemente restritas da população total, às proporções de indivíduos que responderam nunca ter ido ao dentista na vida estariam associados diferentes padrões de restrição do acesso a serviços odontológicos, associados, independentemente da faixa 
etária, a desigualdades de natureza sócio-econômica. Tal suposição é mais plausível quando referida a idades correspondentes a tempos de exposição ao risco de desenvolvimento de problemas de saúde bucal suficientemente longos, como para as faixas de idade selecionadas.

Em pesquisas como a PNAD, é vital considerar as possíveis fontes de erros que interferem nos resultados finais. Além dos potenciais erros sistemáticos já destacados, cabe mencionar a importância dos erros aleatórios, associados ao processo de amostragem. Quanto menor o erro amostral, expresso pelos coeficientes de variação, menor a chance de erro das estimativas 18 . As limitações dos dados da PNAD para a análise das desigualdades sociais no acesso a serviços de saúde em âmbito estadual foram evidenciadas em estudo baseado nos suplementos saúde dos anos de 1998 e 2003. Tais limitações se deveram aos elevados coeficientes de variação das estimativas referentes a níveis de agregação menores que o nacional, especialmente quando envolvendo estimativas de tamanho reduzido ${ }^{41}$. Esse aspecto é particularmente importante quando se observam as OR ajustadas, pois, dado o conjunto de variáveis consideradas nas análises múltiplas, a menor célula de um cruzamento dificilmente ficaria dentro do limite desejado. Para contornar problemas como esse, de forma a assegurar níveis satisfatórios de precisão das estimativas, seria necessário um grande volume de observações, maior do que o disponível. Nesse sentido, os resultados por ora apresentados devem ser interpretados como indicativos de tendências nos sentidos apontados.

\section{Resumo}

O objetivo deste estudo foi analisar o acesso a e a utilização de serviços odontológicos no Estado do Rio de Janeiro, Brasil, em 1998, a partir da Pesquisa Nacional por Amostra de Domicílios (PNAD 1998). A população de estudo foi composta por 7.756 indivíduos subdivididos em três estratos etários (15 a 19, 35 a 44 e 65 a 74 anos). A avaliação do acesso baseou-se na pergunta sobre a última vez que o entrevistado foi ao dentista. O fato de um indivíduo nunca ter ido ao dentista na vida foi considerado como indicativo de restrição de acesso, analisado segundo variáveis demográficas, sócio-econômicas e epidemiológicas. As proporções de indivíduos que nunca consultaram dentista foram $7,6 \%, 1,8 \%$ e 2,6\% respectivamente, entre os jovens, adultos e idosos. Os resultados sugerem que indivíduos menos favorecidos em termos sócio-econômicos apresentavam maior limitação de acesso, ou seja, os menos escolarizados (jovens e adultos), com menor renda per capita (jovens e idosos) e com menor posse de eletrodomésticos (adultos). Entre os jovens e os adultos, a posse de plano de saúde mostrou-se inversamente associada à restrição de acesso.

Assistência Odontológica; Acesso aos Serviços de Saúde; Desigualdades em Saúde

\section{Colaboradores}

A. L. D. Manhães desenvolveu a revisão bibliográfica, a análise dos dados, a organização e a redação do texto. A. J. L. Costa participou na análise dos dados e na redação da versão final do manuscrito. 


\section{Referências}

1. Penchansky R, Thomas W. The concept of access: definition and relationship to consumer satisfaction. Med Care 1981; 19:127-40.

2. Frenk J. El concepto y la medición de accesibilidad. Salud Pública Méx 1985; 27:438-53.

3. Travassos C, Martins M. Uma revisão sobre os conceitos de acesso e utilização de serviços de saúde. Cad Saúde Pública 2004; 20 Suppl 2:S190-8.

4. Pereira MG. Epidemiologia: teoria e prática. Rio de Janeiro: Editora Guanabara Koogan; 1995.

5. Andersen R, Newman JF. Societal and individual determinants of medical care utilization in the United States. Milbank Mem Fund Q 1973; 51:95124.

6. Gilbert HG, Branch LG, Longmate J. Dental care use by U.S. veterans eligible for VA care. Soc Sci Med 1993; 36:361-70.

7. Gilbert HG, Duncan RP, Heft MW, Coward RT. Dental health attitudes among dentate black and white adults. Med Care 1997; 35:255-71.

8. Locker D, Clarke M. Geographic variations in dental services provided to older adults in Ontario, Canada. Community Dent Oral Epidemiol 1999; 27:275-82.

9. Ryan SA, Millstein SG, Greene B, Irwin CE. Utilization of ambulatory health services by urban adolescents. J Adolesc Health 1996; 18:192-202.

10. Swank ME, Vernon SW, Lairson DR. Patterns of preventive dental behavior. Publ Health Rep 1986; 101:175-84.

11. Tennstedt SL, Brambilla DL, Jette AM, McGuire SM. Understanding dental service use by older adults sociobehavioral factors $v s$. need. J Public Health Dent 1994; 54:211-9.

12. Brown LJ, Lazar V. Dental care utilization: how satured is the patient market? J Am Dent Assoc 1999; 130:573-80.

13. Douglass CW, Cole KO. Utilization of dental services in the United States. J Dent Educ 1979; 43: 223-38.

14. Honkala E, Kuusela S, Rimpelã A, Rimpelã M, Jokela J. Dental services utilization between 1977 and 1995 by Finnish adolescents of different socioeconomic levels. Community Dent Oral Epidemiol 1997; 25:385-90.

15. Schwarz E. Changes in demand for dental care among Danish adults, 1975-90. Acta Odontol Scand 1996; 54:36-43.

16. Ministério da Saúde. Levantamento epidemiológico em saúde bucal: cárie dental, capitais, 1996. http://tabnet.datasus.gov.br/cgi/sbucal/ sbdescr.htm (acessado em 06/Fev/2004).

17. Bertoldi DA, Barros DJA. Desigualdade na utilização e no acesso a serviços odontológicos: uma avaliação em nível nacional. Ciênc Saúde Coletiva 2002; 74:709-17.

18. Instituto Brasileiro de Geografia e Estatística. Acesso e utilização de serviços de saúde: 1998. Rio de Janeiro: Instituto Brasileiro de Geografia e Estatística; 2000
19. Ministério da Saúde. Projeto SB Brasil 2003: condições de saúde bucal da população brasileira 20022003. Resultados principais. Brasília: Ministério da Saúde; 2004.

20. Strayer MS, Kuthy RA, Caswell RJ, Moeschberger ML. Predictors of dental use for low-income, urban elderly persons upon removal of financial barriers. Gerontologist 1997; 37:110-6.

21. Holtzman JM, Berkey DB, Mann J. Predicting utilization of dental services by the aged. J Public Health Dent 1990; 50:164-71.

22. Dolan TA, Corey CR, Freeman HE. Older Americans' access to oral health care. J Dent Educ 1988; 52:637-42.

23. Matos DL, Giatti L, Lima-Costa MF. Fatores sócio-demográficos associados ao uso de serviços odontológicos entre idosos brasileiros: um estudo baseado na Pesquisa Nacional por Amostra de Domicílios. Cad Saúde Pública 2004; 20:1290-7.

24. Hobdell M, Petersen PE, Clarkson J, Johnson N. Global goals for oral health 2020. Int Dent J 2003; 53:285-8.

25. Federation Dentaire Internationale. Global goals for oral health in the year 2000. Int Dental J 1982; 32:74-7.

26. World Health Organization. Formulating strategies for health for all by year 2000. Geneva: World Health Organization; 1997.

27. Hosmer DW, Lemeshow S. Applied logistic regression. 2nd Ed. New York: Johns Wiley and Sons; 2000.

28. Lee ES, Forthofer RN, Lorimor RJ. Analyzing complex data surveys. Newbury Park: Sage Publications; 1989.

29. Furlani PA. Influências dos aspectos sociais e culturais na prevalência da cárie dentária em escolares de Jaraguá do Sul [Monografia de Especialização]. Curitiba: Universidade Federal do Paraná; 1993.

30. Martins MD, Araújo RGD, Veloso NF. Avaliação das necessidades de tratamento Odontológico de crianças de baixa renda. J Bras Odontopediatr Odontol Bebê 1999; 2:132-6.

31. Loreto NRM, Sexas ZA, Jardim MC, Brito RL. Cárie dentária no Brasil: alguns aspectos sociais, políticos e econômicos. Rev ABO Nac 2000, 8:45.

32. Matos DL, Lima-Costa MFF, Guerra HL, Marcenes W. Projeto Bambuí: estudo de base populacional dos fatores associados com o uso regular de serviços odontológicos em adultos. Cad Saúde Pública 2001; 17:661-8.

33. Pinheiro RS, Torres TZG. Uso de serviços odontológicos entre os estados do Brasil. Ciênc Saúde Coletiva 2006; 11:999-1010.

34. Gift HC, Newman JF. How older adults use oral health care services: results of a national health interview survey. J Am Dent Assoc 1993; 124:89-93.

35. Pinheiro RS, Viacava F, Travassos C, Brito AS. Gênero, morbidade, acesso e utilização de serviços de saúde no Brasil. Ciênc Saúde Coletiva 2002; 7:687-707. 
36. Todorov A, Kirchner, C. Bias in proxie's reports of disability: data from the National Health Interview Survey on Disability. Am J Public Health 2000; 90:1248-53.

37. McGrath C, Bedi R, Dhawan N. Factors influencing older people's self reported use of dental services in the UK. Gerodontology 1999; 16:97-102.

38. Höloe LA, Tronstad L. The use of dental services among Norwegian adults in 1973. Community Dent Oral Epidemiol 1975; 3:120-5.

39. Locker D, Ford J. Evaluation of an area-based measure as an indicator of inequalities in oral health. Community Dent Oral Epidemiol 1994; 22:80-5.
40. Davenport C, Elley K, Salas C, Taylor-Weetman CL, Fry-Smith A, Bryan S. The clinical effectiveness and cost-effectiveness of routine dental checks: a systematic review and economic evaluation. Health Technol Assess 2003; 7:iii-v, 1-127.

41. Travassos C, Oliveira EXG, Viacava F. Desigualdades geográficas e sociais no acesso aos serviços de saúde no Brasil: 1998 e 2003. Ciênc Saúde Coletiva 2006; 11:975-86.

Recebido em 02/Mar/2006

Versão final reapresentada em 15/Mai/2007

Aprovado em 05/Jun/2007 